\title{
EFEITO DA TERMORRETIFICAÇÃO NAS PROPRIEDADES FÍSICAS E QUÍMICAS DA MADEIRA DE Pinus caribaea
}

\author{
Dallyene da Silva Poubel ${ }^{1 *}$, Rosilei Aparecida Garcia ${ }^{1}$, Wanessa Aparecida dos Santos ${ }^{1}$, \\ Gisely de Lima Oliveira ${ }^{1}$, Heber dos Santos Abreu ${ }^{1}$
}

*Autora para correspondência: dallyenepoubel@hotmail.com

RESUMO: Conduziu-se este estudo, com o objetivo de avaliar o efeito da termorretificação nas propriedades físicas (densidade aparente, teor de umidade de equilíbrio, perda de massa, contração e inchamento lineares e volumétricos) e na composição química da madeira normal (lado oposto) e de compressão de Pinus caribaea Morelet. Amostras de 25 x 25 x $50 \mathrm{~mm}$ foram termorretificadas sob duas temperaturas: 200 e $220^{\circ} \mathrm{C}$. Os resultados indicaram uma redução no teor de umidade de equilíbrio e nos inchamentos e contrações após a termorretificação, independente do tipo de lenho. A madeira de compressão apresentou maior inchamento longitudinal que a madeira normal, entretanto, após a termorretificação, as melhorias foram similares entre os dois tipos de lenho. As madeiras termorretificadas a 200 e $220^{\circ} \mathrm{C}$ apresentaram perdas de massa de 9,94 e 21,03 \%, respectivamente. A termorretificação promoveu um aumento relativo no teor de lignina e uma redução nos teores de holocelulose e $\alpha$-celulose, indicando a ocorrência de degradação térmica desses componentes.

Palavras-chave: Estabilidade dimensional, madeira de compressão, modificação térmica.

\section{EFFECT OF THE HEAT TREATMENT ON PHYSICAL AND CHEMICAL PROPERTIES OF Pinus caribaea WOOD}

\begin{abstract}
This study evaluated the effect of the heat treatment on physical properties (density, equilibrium moisture content, mass loss, volumetric and linear swelling and shrinkage) and chemical composition of normal (opposite side) and compression wood of Pinus caribaea Morelet. Samples of $25 \times 25 \times 50 \mathrm{~mm}$ were heat-treated under two different temperatures: 200 and $220^{\circ} \mathrm{C}$. The results indicated a reduction in equilibrium moisture content and swelling and shrinkage following heat treatment, independent of wood type. The compression wood showed higher longitudinal swelling than normal one, however, after heat treatment, the improvements were similar between both wood types. Samples heat-treated at 200 and $220^{\circ} \mathrm{C}$ had mass losses of 9.94 and $21.03 \%$, respectively. The heat treatment promoted a relative increase in lignin content and a decrease in holocellulose and $\alpha$-cellulose contents, indicating the occurrence of thermal degradation of these components.
\end{abstract}

Key words: Dimensional stability, compression wood, thermal modification.

\section{INTRODUÇÃO}

Um dos maiores desafios para os pesquisadores na área de tecnologia da madeira tem sido encontrar soluções para a instabilidade dimensional e durabilidade natural da madeira. Recentemente, vários trabalhos têm demonstrado a melhoria dessas propriedades quando a madeira é tratada termicamente (GARCIA et al., 2012; KAMDEM et al., 2002). Atualmente, a madeira tratada termicamente vem sendo comercializada, principalmente na Europa, Estados Unidos e Canadá, onde alguns processos foram patenteados, sendo eles: Retification ${ }^{\circledR}$ (França), Bois Perdure $^{\circledR}$ (França e Canadá), Oil Heat Treatment-OHT ${ }^{\circledR}$ (Alemanha), Plato ${ }^{\circledR}$ Wood (Países Baixos), ThermoWood ${ }^{\circledR}$ (Finlândia, Suiça e Canadá) e Westwood (Estados Unidos, Canadá e Rússia) (MILITZ, 2002; RAPP, 2001). As principais diferenças entre esses tratamentos estão relacionadas ao teor de umidade inicial da madeira, ao número de etapas do processo, à utilização de oxigênio ou nitrogênio, à presença ou ausência de vapor, ao processo seco ou úmido e à utilização de óleos (MILITZ, 2002).

Normalmente, os tratamentos térmicos são realizados com temperaturas de até $280{ }^{\circ} \mathrm{C}$ e durações variando de 15 minutos a 24 horas, dependendo do processo, do tamanho da amostra, do teor de umidade da madeira, da espécie em função de sua composição química e estrutura anatômica e das características desejadas ao produto final (KAMDEM et al., 2002).

No Brasil, o tratamento térmico da madeira tem sido realizado a temperaturas próximas de $200^{\circ} \mathrm{C}$, processo denominado de termorretificação, e aplicado para algumas espécies nativas, tais como: Simarouba amara (marupá), Sextonia rubra (louro-vermelho) e Cariniana micrantha (jequitibá-rosa) e, principalmente, para as espécies exóticas, tais como Eucalyptus spp. e Pinus spp. (AZEVEDO; QUIRINO, 2006; BORGES; QUIRINO, 2004; BRITO et al., 2006; CALONEGO, 2009; GARCIA et al., 2012; GOUVEIA, 2008; PALERMO, 2010; PINCELLI et al., 2002).

${ }^{1}$ Universidade Federal Rural do Rio de Janeiro - Seropédica, Rio de Janeiro, Brasil

Cerne, Lavras, v. 19, n. 3, p. 391-398, jul./set. 2013 
Além da maior estabilidade dimensional e durabilidade natural, a madeira termorretificada apresenta outras características tais como: cor escura, perda de massa, alterações químicas, maior capacidade de isolação térmica e não requer a utilização de preservativos químicos, o que representa uma vantagem sob o ponto de vista ambiental (KAMDEM et al., 2002). Por outro lado, os tratamentos de termorretificação conferem à madeira uma baixa resistência mecânica e menor rigidez quando realizados em condições severas de temperatura e tempo, o que pode comprometer a aplicação do material para fins estruturais (CALONEGO, 2009; GARCIA et al., 2012; UNSAL et al., 2003).

A madeira de Pinus spp. tem sido uma das mais estudadas no processo de termorretificação, a fim de agregar maior valor ao produto final, já que a mesma apresenta características indesejáveis, tais como: baixa durabilidade, baixa estabilidade dimensional e cor pálida (FINNISH THERMOWOOD ASSOCIATION, 2003; RAPP, 2001). Segundo a International ThermoWood Association (2012), $51 \%$ da madeira tratada pelo processo ThermoWood $^{\circledR}$, em 2011, foi representada pelo gênero Pinus. Entretanto, a presença de madeira juvenil e de defeitos naturais na madeira tais como nós e madeira de compressão podem gerar um comportamento diferente face ao tratamento e seus efeitos nas propriedades da madeira termorretificada são pouco conhecidos. Segundo Boone e Chudnoff (1972), a madeira de Pinus caribaea apresenta uma alta incidência de madeira de compressão mesmo em árvores relativamente retas e esta apresenta características anatômicas, físicas e químicas diferentes da madeira normal. A madeira de compressão apresenta maior densidade e sua presença pode ocasionar contrações longitudinais maiores que na madeira normal, gerando maior ocorrência de empenamentos (WALKER, 2006).

Nesse contexto, neste estudo, objetivou-se avaliar o efeito da termorretificação nas propriedades físicas (densidade aparente, teor de umidade de equilíbrio, perda de massa, contração e inchamento lineares e volumétricos) e na composição química da madeira normal (lado oposto) e de compressão de Pinus caribaea Morelet.

\section{MATERIAL E MÉTODOS}

\subsection{Matéria prima e tratamento de termorretificação}

Foram utilizadas árvores de Pinus caribaea Morelet com 25 anos de idade, coletadas de um plantio com espaçamento de $2 \times 3 \mathrm{~m}$ localizado no Campus da Universidade Federal Rural do Rio de Janeiro (UFRRJ), Seropédica, RJ. Das árvores, foram obtidos quatro discos de $10 \mathrm{~cm}$ de espessura, retirados a 1,30 m do solo (diâmetro a altura do peito - DAP). De cada disco foram retiradas 24 amostras de $25 \times 25 \times 50 \mathrm{~mm}$ (espessura x largura x comprimento), perfeitamente orientadas nos planos transversal, longitudinal, tangencial e radial, sendo 12 amostras de madeira normal (lado oposto) e 12 de madeira de compressão.

As amostras foram secas em estufa a $60{ }^{\circ} \mathrm{C}$ e acondicionadas em câmara climática a $20{ }^{\circ} \mathrm{C}$ e $65 \%$ de umidade relativa (UR) até atingir o teor de umidade de equilíbrio para posterior tratamento de termorretificação.

As amostras foram termorretificadas, sob duas diferentes condições de temperatura $\left(200\right.$ e $\left.220^{\circ} \mathrm{C}\right)$ durante 2 horas em uma estufa de circulação de ar disponível no Laboratório de Secagem da Madeira do Departamento de Produtos Florestais (DPF), Instituto de Florestas (IF), UFRRJ. Após o tratamento, as amostras foram reacondicionadas em câmara climática a $20^{\circ} \mathrm{C}$ e $65 \%$ UR, até atingirem o teor de umidade de equilíbrio e utilizadas para a determinação das propriedades físicas e caracterização química.

As propriedades físicas determinadas foram: a densidade aparente, teor de umidade de equilíbrio, contrações (volumétrica e lineares) e inchamentos (volumétrico e lineares) das madeiras normal e de compressão termorretificadas e não termorretificadas e a perda de massa após o tratamento de termorretificação. Para a caracterização química, determinaram-se os teores de extrativos, de lignina de Klason, de holocelulose e de $\alpha$-celulose.

\subsection{Determinação das propriedades físicas da madeira}

A densidade aparente e teor de umidade de equilíbrio das madeiras não termorretificadas e termorretificadas foram determinadas de acordo com a norma ASTM D 2395 (AMERICAN SOCIETY FOR TESTING AND MATERIALS - ASTM, 1999b).

As variações dimensionais da madeira (contrações e inchamentos volumétricos e lineares) foram determinadas de acordo com a norma ASTM D 143 (ASTM, 1999a).

A porcentagem da perda de massa foi determinada ao dividir a perda de massa sofrida pela amostra após a termorretificaçao pela massa inicial da mesma.

\subsection{Caracterização química da madeira}

A caracterização química das madeiras normal e de compressão termorretificadas e não termorretificadas foi realizada de acordo com os métodos de análises químicas descritos por Abreu et al. (2006) que foram adaptados das normas ASTM D 1105 e D 1106. Foram determinados os

Cerne, Lavras, v. 19, n. 3, p. 391-398, jul./set. 2013 
teores de extrativos, de lignina de Klason, de holoceluloses e de $\alpha$-celulose. Para os extrativos, foram obtidos os teores após a extração em um extrator de tipo Soxhlet, durante 24, horas com três solventes orgânicos em ordem crescente de polaridade: cicloexano, acetato de etila e metanol.

\subsection{Análises estatísticas}

O delineamento experimental utilizado foi o inteiramente casualizado composto por seis tratamentos: lenho normal não termorretificado e termorretificado a 200 e $200{ }^{\circ} \mathrm{C}$ e lenho de compressão não termorretificado e termorretificado a 200 e $200{ }^{\circ} \mathrm{C}$. Foram utilizadas 12 amostras para cada tratamento, totalizando 72 observações.

Para as análises químicas, foram utilizadas duas repetições de cada tratamento para a determinação do teor de extrativos e três repetições para a determinação dos teores de lignina de Klason, de holoceluloses e de $\alpha$-celulose.

Os dados foram submetidos a uma análise de variância e quando houve diferença significativa entre os tratamentos aplicou-se o teste de Duncan, a fim de verificar as diferenças entre as médias dos tratamentos.

\section{RESULTADOS E DISCUSSÃO}

\subsection{Propriedades físicas}

Os resultados obtidos para as propriedades físicas das madeiras normal e de compressão termorretificadas e não termorretificadas (controle) são apresentados na Tabela 1. As análises estatísticas indicaram diferenças significativas entre os tratamentos para todas as propriedades físicas estudadas. Comparando a madeira normal com a madeira de compressão não termorretificada, observaram-se diferenças significativas somente no inchamento tangencial (maior na madeira normal) e no inchamento longitudinal (maior na madeira de compressão), enquanto que, para as demais propriedades físicas, não foram obtidas diferenças significativas entre os tipos de lenho (Tabela 1). O maior inchamento longitudinal na madeira de compressão pode ser explicado pelo comprimento dos traqueídeos. Segundo Tomazello Filho e Silva (1987), a madeira de compressão apresenta traqueídeos mais curtos, resultando em maiores variações dimensionais no sentido longitudinal.

Tabela 1 - Médias, desvio padrão e valor F das propriedades físicas da madeira termorretificada de Pinus caribaea.

Table 1 - Means, standard deviation and F value of the physical properties of the heat-treated Pinus caribaea wood.

\begin{tabular}{|c|c|c|c|c|c|c|c|c|c|c|c|c|}
\hline $\begin{array}{l}\text { Tipo de } \\
\text { lenho }\end{array}$ & $\begin{array}{c}\text { Temperatura } \\
\left({ }^{\circ} \mathrm{C}\right)\end{array}$ & $\begin{array}{c}\text { Densidade } \\
\left(\mathrm{g} / \mathrm{cm}^{3}\right)\end{array}$ & $\begin{array}{l}\text { TUE } \\
(\%)\end{array}$ & $\begin{array}{l}\beta_{\mathrm{V}} \\
(\%)\end{array}$ & $\begin{array}{c}\beta_{\mathrm{T}} \\
(\%)\end{array}$ & $\begin{array}{l}\beta_{\mathrm{R}} \\
(\%)\end{array}$ & $\begin{array}{l}\beta_{\mathrm{L}} \\
(\%)\end{array}$ & $\begin{array}{c}\alpha_{\mathrm{V}} \\
(\%)\end{array}$ & $\begin{array}{c}\alpha_{\mathrm{T}} \\
(\%)\end{array}$ & $\begin{array}{l}\alpha_{R} \\
(\%)\end{array}$ & $\begin{array}{c}\alpha_{L} \\
(\%)\end{array}$ & $\begin{array}{l}\mathrm{PM} \\
(\%)\end{array}$ \\
\hline \multirow{3}{*}{ Normal } & Cont1 & $(0,03)$ & $\begin{array}{c}13,98 \mathrm{ab} \\
(0,43)\end{array}$ & $\begin{array}{c}14,33 \mathrm{a} \\
(1,81)\end{array}$ & $\begin{array}{l}8,89 \text { a } \\
(1,05)\end{array}$ & $\begin{array}{c}5,56 \mathrm{ab} \\
(1,07)\end{array}$ & $\begin{array}{c}1,10 \mathrm{ab} \\
(0,16)\end{array}$ & $\begin{array}{c}18,83 \mathrm{a} \\
(2,80)\end{array}$ & $\begin{array}{l}9,49 \mathrm{a} \\
(1,58)\end{array}$ & $\begin{array}{l}7,49 \mathrm{a} \\
(1,16)\end{array}$ & $\begin{array}{l}1,49 \mathrm{~b} \\
(0,13)\end{array}$ & - \\
\hline & 200 & $\begin{array}{c}0,528 \mathrm{~b} \\
(0,03)\end{array}$ & $\begin{array}{c}11,66 \mathrm{c} \\
(0,88)\end{array}$ & $\begin{array}{c}10,51 b c \\
(1,66)\end{array}$ & $\begin{array}{c}5,13 \text { bc } \\
(0,73)\end{array}$ & $\begin{array}{l}5,23 \mathrm{~b} \\
(0,49)\end{array}$ & $\begin{array}{c}0,77 \mathrm{bc} \\
(0,13)\end{array}$ & $\begin{array}{c}12,10 \mathrm{bc} \\
(1,83)\end{array}$ & $\begin{array}{c}5,41 \mathrm{bc} \\
(1,01)\end{array}$ & $\begin{array}{c}5,25 \mathrm{ab} \\
(0,36)\end{array}$ & $\begin{array}{l}1,18 \mathrm{~b} \\
(0,18)\end{array}$ & $\begin{array}{l}10,41 \mathrm{~b} \\
(1,00)\end{array}$ \\
\hline & 220 & $\begin{array}{c}0,517 \mathrm{bc} \\
(0,03)\end{array}$ & $\begin{array}{l}8,36 \mathrm{~d} \\
(0,27)\end{array}$ & $\begin{array}{c}8,39 \mathrm{~cd} \\
(0,96)\end{array}$ & $\begin{array}{l}4,56 \mathrm{c} \\
(0,85)\end{array}$ & $\begin{array}{l}4,33 \mathrm{c} \\
(0,33)\end{array}$ & $\begin{array}{l}0,44 \mathrm{c} \\
(0,09)\end{array}$ & $\begin{array}{l}9,31 \mathrm{~cd} \\
(1,04)\end{array}$ & $\begin{array}{l}4,79 \mathrm{c} \\
(0,94)\end{array}$ & $\begin{array}{c}4,79 \text { bc } \\
(0,94)\end{array}$ & $\begin{array}{l}0,56 \mathrm{~b} \\
(0,08)\end{array}$ & $\begin{array}{c}19,48 \mathrm{a} \\
(2,00)\end{array}$ \\
\hline \multirow{3}{*}{ Compressão } & Controle & $\begin{array}{c}0,578 \mathrm{a} \\
(0,03)\end{array}$ & $\begin{array}{c}14,96 \mathrm{a} \\
(0,79)\end{array}$ & $\begin{array}{c}12,37 \mathrm{ab} \\
(1,28)\end{array}$ & $\begin{array}{c}7,63 \mathrm{ab} \\
(1,05)\end{array}$ & $\begin{array}{l}6,15 \mathrm{a} \\
(0,73)\end{array}$ & $\begin{array}{l}2,54 \mathrm{a} \\
(0,30)\end{array}$ & $\begin{array}{c}16,82 \mathrm{ab} \\
(1,33)\end{array}$ & $\begin{array}{l}7,44 \mathrm{~b} \\
(1,22)\end{array}$ & $\begin{array}{c}6,55 \mathrm{abc} \\
(0,82)\end{array}$ & $\begin{array}{l}2,47 \mathrm{a} \\
(0,22)\end{array}$ & - \\
\hline & 200 & $\begin{array}{c}0,535 \mathrm{~b} \\
(0,02)\end{array}$ & $\begin{array}{c}11,88 \mathrm{bc} \\
(0,53)\end{array}$ & $\begin{array}{c}9,67 \mathrm{~cd} \\
(1,28)\end{array}$ & $\begin{array}{c}5,09 \mathrm{bc} \\
(0,62)\end{array}$ & $\begin{array}{l}4,36 \mathrm{c} \\
(0,91)\end{array}$ & $\begin{array}{c}1,20 \mathrm{bc} \\
(0,04)\end{array}$ & $\begin{array}{c}11,24 \mathrm{~cd} \\
(0,61)\end{array}$ & $\begin{array}{l}5,36 \mathrm{bc} \\
(0,68)\end{array}$ & $\begin{array}{c}5,06 \text { bc } \\
(0,26)\end{array}$ & $\begin{array}{l}1,21 \mathrm{~b} \\
(0,16)\end{array}$ & $\begin{array}{l}9,46 \mathrm{~b} \\
(1,11)\end{array}$ \\
\hline & 220 & $\begin{array}{c}0,502 \mathrm{c} \\
(0,02)\end{array}$ & $\begin{array}{l}9,19 \mathrm{~d} \\
(0,64)\end{array}$ & $\begin{array}{l}7,92 \mathrm{~d} \\
(1,12)\end{array}$ & $\begin{array}{l}4,24 \mathrm{c} \\
(0,77)\end{array}$ & $\begin{array}{l}4,11 \mathrm{c} \\
(0,79)\end{array}$ & $\begin{array}{c}1,13 \text { bc } \\
(0,15)\end{array}$ & $\begin{array}{l}8,16 \mathrm{~d} \\
(0,91)\end{array}$ & $\begin{array}{l}4,04 \mathrm{c} \\
(0,56)\end{array}$ & $\begin{array}{l}1,57 \mathrm{c} \\
(0,08)\end{array}$ & $\begin{array}{l}1,14 \mathrm{~b} \\
(0,15)\end{array}$ & $\begin{array}{c}22,57 \mathrm{a} \\
(2,57)\end{array}$ \\
\hline Valor F & & $\begin{array}{c}7,65^{* *} \\
\operatorname{Pr}>\mathrm{F} \\
<0,0001\end{array}$ & $\begin{array}{c}4,28 * * \\
\operatorname{Pr}>\mathrm{F} \\
<0,0001\end{array}$ & $\begin{array}{c}4,20 * * \\
\operatorname{Pr}>\mathrm{F} \\
<0,0001\end{array}$ & $\begin{array}{c}2,75^{* *} \\
\operatorname{Pr}>\mathrm{F} \\
0,0028\end{array}$ & $\begin{array}{c}2,92 * * \\
\operatorname{Pr}>\mathrm{F} \\
0,0032\end{array}$ & $\begin{array}{c}2,68 * * \\
\operatorname{Pr}>\mathrm{F} \\
0,0036\end{array}$ & $\begin{array}{c}4,16 * * \\
\operatorname{Pr}>\mathrm{F} \\
<0,0001\end{array}$ & $\begin{array}{c}3,75^{* *} \\
\operatorname{Pr}>\mathrm{F} \\
0,0001\end{array}$ & $\begin{array}{c}2,12^{*} \\
\operatorname{Pr}>\mathrm{F} \\
0,0211\end{array}$ & $\begin{array}{c}2,61 * * \\
\operatorname{Pr}>F \\
0,0044\end{array}$ & $\begin{array}{c}6,01 * * \\
\operatorname{Pr}>\mathrm{F} \\
<0,0001\end{array}$ \\
\hline
\end{tabular}

TUE: teor de umidade de equilíbrio. $\beta_{\mathrm{V}}$ : contração volumétrica. $\beta_{\mathrm{T}}$ : contração tangencial. $\beta_{\mathrm{R}}$ : contração radial. $\beta_{\mathrm{L}}$ : contração longitudinal. $\alpha_{\mathrm{V}}$ : inchamento volumétrico. $\alpha_{\mathrm{T}}$ : inchamento tangencial. $\alpha_{\mathrm{R}}$ : inchamento radial. $\alpha_{\mathrm{L}}$ : inchamento longitudinal. PM: perda de massa após o tratamento de termorretificação. ** Significativo à $99 \%$ de probabilidade. * Significativo à $95 \%$ de probabilidade. Médias com a mesma letra não são estatisticamente diferentes entre si segundo o teste de Duncan ao nível de $5 \%$ de significância.

Cerne, Lavras, v. 19, n. 3, p. 391-398, jul./set. 2013 
A termorretificação causou uma redução significativa na densidade aparente $(\mathrm{F}=7,65, \operatorname{Pr}>\mathrm{F}<0,001)$, independente do tipo de lenho (normal x compressão). A menor densidade aparente foi observada para as madeiras termorretificadas a $220^{\circ} \mathrm{C}$.

Os teores de umidade de equilíbrio das madeiras termorretificadas (normal e compressão) foram significativamente menores $(\mathrm{F}=4,28, \operatorname{Pr}>\mathrm{F}<0,001)$ que aqueles obtidos para as madeiras não termorretificadas. Resultados similares foram encontrados por Borges e Quirino (2004) para a madeira termorretificada de Pinus caribaea var. hondurensis.

Para a contração volumétrica, as madeiras termorretificadas apresentaram valores significativamente menores $(F=4,20, \operatorname{Pr}>F<0,001)$ que os obtidos para as madeiras não termorretificadas, entretanto, não foram observadas diferenças significativas entre as temperaturas de tratamento.

Para a contração tangencial, no caso da madeira de compressão, somente a madeira termorretificada a $220{ }^{\circ} \mathrm{C}$ apresentou reduções significativas $(\mathrm{F}=2,75, \operatorname{Pr}>\mathrm{F}<$ $0,001)$ quando comparada ao controle, enquanto para madeira normal ambas as temperaturas apresentaram reduções, não diferenciando entre si.

Para a contração radial, para a madeira normal, somente a madeira termorretificada a $220^{\circ} \mathrm{C}$ apresentou reduções significativas $(F=2,92, \operatorname{Pr}>F<0,0032)$ quando comparada ao controle, enquanto que, para a madeira de compressão, ambas as temperaturas apresentaram reduções, não diferenciando entre si.

Para a contração longitudinal, no caso da madeira de compressão, a termorretificação causou reduções significativas $(F=2,68, \operatorname{Pr}>F<0,001)$ independente da temperatura, enquanto que, para a madeira normal, somente o tratamento a $220{ }^{\circ} \mathrm{C}$ apresentou reduções significativas em relação ao controle.

Para o inchamento volumétrico, tanto para a madeira normal quanto para a madeira de compressão, a termorretificação causou reduções significativas $(\mathrm{F}=$ $4,16, \operatorname{Pr}>\mathrm{F}<0,001)$, independente das temperaturas de tratamento.

Para o inchamento tangencial, no caso da madeira normal, a termorretificação promoveu reduções significativas $(F=3,75, \operatorname{Pr}>F<0,001)$ para ambas as temperaturas, no entanto, para madeira de compressão, houve redução significativa somente a $220^{\circ} \mathrm{C}$.

No inchamento radial, para madeira de compressão, não foram encontradas diferenças significativas entre a madeira termorretificada e não termorretificada. Entretanto, para a madeira normal, o tratamento a $220^{\circ} \mathrm{C}$ reduziu significativamente $\mathrm{o}$ inchamento $\operatorname{radial}(\mathrm{F}=2,12$, $\operatorname{Pr}>\mathrm{F}<0,005)$

No inchamento longitudinal, a termorretificação afetou apenas a madeira de compressão, causando uma redução significativa $(\mathrm{F}=2,61, \operatorname{Pr}>\mathrm{F}<0,001)$, independente da temperatura.

Os resultados obtidos para o teor de umidade de equilíbrio e para as variações dimensionais (inchamentos e contrações) da madeira indicaram uma melhoria significativa na estabilidade dimensional da madeira, após a termorretificação, independente do tipo de lenho (normal x compressão).

Comparando as variações dimensionais nas diferentes direções estruturais, observa-se que os valores de contração e inchamento na direção tangencial são maiores que na direção radial, os quais, por sua vez, são maiores que na direção longitudinal. Isso ocorre, em decorrência da anisotropia da madeira. Segundo Skaar (1988), as variações dimensionais no sentido transversal (tangencial e radial) são maiores que no sentido longitudinal, já que a maioria das células está orientada longitudinalmente. No sentido radial, as variações dimensionais são menores que no sentido tangencial por causa da orientação transversal dos raios e da presença de pontoações areoladas, predominante nas paredes radiais das traqueídeos, causando um desvio das microfibrilas de celulose na camada S2.

Os resultados indicaram perdas de massa significativas após a termorretificação $(\mathrm{F}=6,01, \operatorname{Pr}>\mathrm{F}<$ 0,001 ), sendo maiores para as temperaturas mais elevadas, independentemente do tipo de lenho (normal x compressão). A termorretificação a $200{ }^{\circ} \mathrm{C}$ causou uma perda de massa de $9,94 \%$, enquanto a $220{ }^{\circ} \mathrm{C}$, essa perda foi de $21,03 \%$. A perda de massa ocorre, principalmente, pela perda de água presente nas paredes celulares da madeira e pela perda de substâncias voláteis, entretanto, de acordo com alguns autores (BORREGA; KÄRENLAMPI, 2008; CHARANI et al., 2007; ESTEVES et al., 2007) essa perda também pode ser explicada pela degradação das hemiceluloses, as quais são os componentes estruturais mais afetados pelo calor e os mais susceptíveis à degradação térmica. Essa degradação é irreversível e vem associada à degradação dos grupos hidroxílicos acessíveis, ou seja, responsáveis pela absorção de água nas paredes celulares da madeira, o que explica o menor teor de umidade de equilíbrio e a maior estabilidade dimensional (menores inchamentos e contrações) da madeira termorretificada. 


\subsection{Propriedades químicas}

Os resultados obtidos para os teores de extrativos, de lignina de Klason, de holocelulose e de $\alpha$-celulose das madeiras normal e de compressão termorretificadas e não termorretificadas são apresentados nas Tabelas 2 e 3 . As análises estatísticas indicaram diferenças significativas entre os tratamentos para todas as propriedades químicas estudadas, com exceção do teor de extrativos. Apesar da termorretificação não afetar significativamente o teor de extrativos, verificou-se um aumento no mesmo para as madeiras termorretificadas a $200{ }^{\circ} \mathrm{C}$ seguido de um decréscimo para as madeiras termorretificadas a $220{ }^{\circ} \mathrm{C}$, independente do tipo de lenho (normal ou compressão). Segundo a Finnish Thermowood Association (2003), a termorretificação a $120-180{ }^{\circ} \mathrm{C}$ produz um aumento no teor de extrativos, enquanto a $230{ }^{\circ} \mathrm{C}$ há uma redução. $\mathrm{O}$ aumento no teor de extrativos a temperaturas mais baixas pode ser explicado pela formação de subprodutos causada pela degradação das hemiceluloses enquanto a redução no teor de extrativos a temperaturas mais elevadas deve-se à volatilização dos extrativos e à polimerização desses subprodutos com outros polímeros da parede celular, fixando-se na madeira (FINNISH THERMOWOOD ASSOCIATION, 2003; KAMDEM et al., 2002).

Comparando a madeira normal e de compressão não termorretificadas, foram observadas diferenças significativas somente nos teores de lignina de Klason. A madeira de compressão apresentou um maior teor de lignina $(27,11 \%$ ) que a madeira normal (23\%) (Tabela 3$)$.

Tabela 2 - Médias, desvio padrão e valor F dos teores de extrativos da madeira termorretificada de Pinus caribaea.

Table 2 -Means, standard deviation and F value of the extractive contents of the heat-treated Pinus caribaea wood.

\begin{tabular}{lccccc}
\hline \multirow{2}{*}{ Tipo de lenho } & Temperatura & \multicolumn{3}{c}{ Teor de extrativos $(\%)$} & Teor total de \\
\cline { 3 - 5 } & $\left({ }^{\circ} \mathrm{C}\right)$ & Cicloexano & Acetato de etila & Metanol & extrativos $(\%)$ \\
\hline \multirow{2}{*}{ Normal } & Controle & $0,87(0,02)$ & $0,55(0,03)$ & $0,38(0,01)$ & $1,80(0,12)$ \\
& 200 & $0,63(0,07)$ & $0,69(0,07)$ & $1,28(0,08)$ & $2,60(0,04)$ \\
& 220 & $0,44(0,06)$ & $0,91(0,17)$ & $1,05(0,14)$ & $2,39(0,10)$ \\
\hline \multirow{2}{*}{ Compressão } & Controle & $0,79(0,12)$ & $0,34(0,05)$ & $0,49(0,01)$ & $1,62(0,11)$ \\
& 200 & $1,23(0,17)$ & $1,22(0,17)$ & $0,28(0,05)$ & $2,73(0,14)$ \\
\hline \multirow{2}{*}{ Valor F } & 220 & $0,33(0,01)$ & $0,53(0,02)$ & $1,25(0,05)$ & $2,11(0,14)$ \\
\hline
\end{tabular}

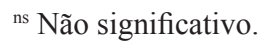

Tabela 3 - Médias, desvio padrão e valor F dos componentes químicos da madeira termorretificada de Pinus caribaea.

Table 3 -Means, standard deviation and F value of the chemical components of the heat-treated Pinus caribaea wood.

\begin{tabular}{lcccc}
\hline Tipo de lenho & $\begin{array}{c}\text { Temperatura } \\
\left({ }^{\circ} \mathrm{C}\right)\end{array}$ & $\begin{array}{c}\text { Teor de lignina } \\
\text { de Klason }(\%)\end{array}$ & $\begin{array}{c}\text { Teor de } \\
\text { holocelulose }(\%)\end{array}$ & $\begin{array}{c}\text { Teor de } \\
\alpha \text {-celulose }(\%)\end{array}$ \\
\hline \multirow{2}{*}{ Normal } & Controle & $23,00 \mathrm{~d}(0,47)$ & $67,03 \mathrm{a}(2,15)$ & $55,90 \mathrm{a}(0,95)$ \\
& 200 & $22,67 \mathrm{~d}(1,41)$ & $55,86 \mathrm{~b}(1,05)$ & $57,05 \mathrm{a}(2,15)$ \\
\hline \multirow{2}{*}{ Compressão } & 220 & $23,00 \mathrm{~d}(1,20)$ & $53,44 \mathrm{~b}(0,39)$ & $44,55 \mathrm{~b}(4,24)$ \\
& Controle & $27,11 \mathrm{c}(0,96)$ & $64,65 \mathrm{a}(2,41)$ & $57,37 \mathrm{a}(1,05)$ \\
\hline \multirow{2}{*}{ Valor F } & 200 & $32,00 \mathrm{~b}(0,47)$ & $55,60 \mathrm{~b}(2,80)$ & $46,55 \mathrm{~b}(6,88)$ \\
& 220 & $36,11 \mathrm{a}(0,38)$ & $46,00 \mathrm{c}(1,74)$ & $38,67 \mathrm{~b}(4,63)$ \\
\hline
\end{tabular}

** Significativo à $99 \%$ de probabilidade. Médias com a mesma letra não são estatisticamente diferentes entre si (Duncan; $\mathrm{p} \geq 0,05)$.

Cerne, Lavras, v. 19, n. 3, p. 391-398, jul./set. 2013 
Esses resultados estão de acordo com aqueles encontrados na literatura por Walker (2006) e Yeh et al. (2006), nos quais os maiores teores de lignina e menores teores de celulose estão presentes na madeira de compressão. Os valores de lignina encontrados para a madeira de Pinus caribaea estão próximos daqueles encontrados para outras espécies de Pinus sp. Por exemplo, Morais et al. (2005) encontraram $25,18 \%$ de lignina de Klason para a madeira de Pinus oocarpa.

A termorretificação afetou o teor de lignina da madeira de compressão, entretanto nenhuma diferença foi constatada entre a madeira normal termorretificada e não termorretificada. As madeiras de compressão termorretificadas apresentaram um aumento significativo $(F=17,39, \operatorname{Pr}>F<0,001)$ no teor de lignina (Tabela 3). Resultados similares foram observados por Brito et al. (2008) e Yildiz et al. (2006). Este aumento no teor de lignina não indica a formação desse polímero durante o tratamento térmico, mas a degradação de outros componentes químicos da madeira, principalmente das hemiceluloses (PONCSÁK et al., 2006), fato que pode ser observado pela redução no teor de holocelulose (Tabela 3). Os teores de holocelulose, tanto na madeira normal quanto na madeira de compressão, foram significativamente menores $(\mathrm{F}=81,32, \operatorname{Pr}>\mathrm{F}<0,0001)$ após a termorretificação.

Para os teores de $\alpha$-celulose, a madeira normal apresentou uma redução significativa somente após a termorretificação a $220^{\circ} \mathrm{C}(\mathrm{F}=7,44, \operatorname{Pr}>\mathrm{F}<0,0027)$, enquanto na madeira de compressão, a termorretificação causou reduções significativas independentemente da temperatura (Tabela 3). Segundo Figueroa e Moraes (2009), a degradação térmica da celulose ocorre entre 200 e $280{ }^{\circ} \mathrm{C}$, sendo esta progressiva e ocorrendo pela despolimerização e desidratação. De acordo com Finnish Thermowood Association (2003), as hemiceluloses são os primeiros polímeros a serem degradados durante a termorretificação. Como as hemiceluloses são os polímeros mais higroscópicos da parede celular, sua degradação causa uma redução no teor de umidade de equilíbrio, entretanto, nesse processo, também ocorre a formação de ácido acético e ácido fórmico, os quais agem como uma catalisador para a despolimerização das microfibrilas de celulose, quebrando-as em cadeias mais curtas.

\section{CONCLUSÕES}

A termorretificação proporcionou uma maior estabilidade dimensional à madeira, apresentando menor teor de umidade de equilíbrio e menores contrações e inchamentos, independente do tipo de lenho (normal x compressão).

A madeira de compressão apresentou maior inchamento longitudinal que a madeira normal, entretanto, após a termorretificação, a melhoria nessa propriedade não foi diferente da madeira normal.

As madeiras termorretificadas apresentaram perda de massa, sendo esta crescente com o aumento da temperatura.

As análises químicas indicaram uma degradação dos polímeros da madeira após a termorretificação, resultando em maiores teores de ligninas e menores teores de holoceluloses e $\alpha$-celulose.

\section{AGRADECIMENTOS}

Os autores agradecem à Fundação Carlos Chagas Filho de Amparo à Pesquisa do Estado do Rio de Janeiro (FAPERJ) e ao Programa de Iniciação Científica da Universidade Federal Rural do Rio de Janeiro (PROIC/ UFRRJ), pelas bolsas de iniciação científica concedidas à primeira e terceira autora, respectivamente.

\section{REFERÊNCIAS}

ABREU, H. S. et al. Métodos de análise em química da madeira. Seropédica: UFRRJ, 2006. 20 p. (Série Técnica Floresta e Ambiente).

AMERICAN SOCIETY FOR TESTING AND MATERIALS. D 143: standard methods of testing small clear specimens of timber. Philadelphia, 1999a. 30 p.

AMERICAN SOCIETY FOR TESTING AND MATERIALS. D 2395: standard test methods for specific gravity of wood and wood-based materials. Philadelphia, 1999. 8 p.

AZEVEDO, A. C. S.; QUIRINO, W. F. Aumento da estabilidade na madeira de eucalipto através de tratamento térmico.

Revista da Madeira, São Paulo, v. 16, n. 98, p. 50-58, 2006.

BOONE, R. S.; CHUDNOFF, M. Compression wood formation and other characteristics of plantation-grown Pinus caribaea. Washington: Institute Tropical Forestry, 1972.

BORGES, L. M.; QUIRINO, W. F. Higroscopicidade da madeira de Pinus caribaea var. hondurensis tratado termicamente. Biomassa \& Energia, Rio de Janeiro, v. 1, n. 2, p. 173-182, 2004.

Cerne, Lavras, v. 19, n. 3, p. 391-398, jul./set. 2013 
BORREGA, M.; KÄRENLAMPI, P. P. Mechanical behavior of heat-treated spruce spruce (Picea abies) wood at constant moisture content and ambient humidity. Holz als Roh-und Werkstoff, Berlin, v. 66, p. 63-69, 2008.

BRITO, O. B. et al. Chemical composition changes in Eucalyptus and Pinus wood submitted to heat treatment. Bioresource Technology, Essex, v. 99, p. 8545-8548, 2008.

BRITO, O. B. et al. Densidade básica e retratibilidade da madeira de Eucalyptus grandis submetida a diferentes temperaturas de termorretificação. Cerne, Lavras, v. 12, n. 2, p. 182-188, 2006.

CALONEGO, F. W. Efeito da termorretificação nas propriedades físicas, mecânicas e na resistência a fungos deterioradores da madeira de Eucalyptus grandis Hill. ex Maiden. 2009. 149 p. Tese (Doutorado em Energia na Agricultura) - Universidade Estadual Paulista "Júlio de Mesquita Filho", Botucatu, 2009.

CHARANI, P. R. et al. Influence of hydrothermal treatment on the dimensional stability of beech wood. Caspian Journal of Environmental Sciences, Guilan, v. 5, n. 2, p. 125-131, 2007.

ESTEVES, B. et al. Influence of steam heating on the properties of pine (Pinus pinaster) and eucalypt (Eucalyptus globulus) wood. Wood Science and Technology, New York, v. 41, n. 3, p. 193-207, 2007.

FIGUEROA, M. J. M.; MORAES, P. D. Comportamento da madeira a temperaturas elevadas. Ambiente Construído, Porto Alegre, v. 9, n. 4, p. 157-174, 2009.

\section{FINNISH THERMOWOOD ASSOCIATION. ThermoWood ${ }^{\circledR}$} handbook. Helsinki, 2003. 66 p.

GARCIA, R. A. et al. Nondestructive evaluation of heattreated Eucalyptus grandis Hill ex Maiden wood using stress wave method. Wood Science and Technology, New York, v. 46, p. 41-52, 2012.

GOUVEIA, F. N. Aplicação de tratamentos térmicos para estabilização colorimétrica de madeiras tropicais. 2008. 130 p. Tese (Doutorado em Ciência Florestal) - Universidade de Brasília, Brasília, 2008.
INTERNATIONAL THERMOWOOD ASSOCIATION. Thermowood production statistics 2012. Disponível em: $<$ http://www.thermowood.fi $>$. Acesso em: 22 out. 2012.

KAMDEM, D. P.; PIZZI, A.; JERMANNAUD, A. Durability of heat-treated wood. Holz als Roh-und Werkstoff, Berlin, v. 60 , p. 1-6, 2002.

MILITZ, R. Heat treatment technologies in Europe: scientific background and technological state-of-art. In: CONFERENCE ON ENHANCING THE DURABILITY OF LUMBER AND ENGINEERED WOOD PRODUCTS, 1., 2002, Orlando. Proceedings... Madison: Forest Products Society, 2002. p. $1-19$.

MORAIS, S. A. L.; NASCIMENTO, E. A.; MELO, D. C. Análise da madeira de Pinus oocarpa parte I: estudo dos constituintes macromoleculares e extrativos voláteis. Revista Árvore, Viçosa, v. 29, n. 3, p. 461-470, maio/jun. 2005.

PALERMO, G. P. M. Propriedades e comportamento tecnológico da madeira de Eucalyptus grandis Hill ex. Maiden visando a sua utilização em produtos de maior valor agregado. 2010. 237 p. Tese (Doutorado em Ciências Ambientais e Florestais) - Universidade Federal Rural do Rio de Janeiro, Seropédica, 2010.

PINCELLI, A. L. P. S. M.; BRITO, J. O.; CORRENTE, J. E. Avaliação da termorretificação sobre a colagem na madeira de Eucalyptus saligna e Pinus caribaea var. hondurensis. Scientia Forestalis, Piracicaba, n. 61, p. 122-132, 2002.

PONCSÁK, S. et al. Effect of high temperature treatment on the mechanical properties of birch (Betula papyrifera). Wood Science and Technology, New York, v. 40, n. 1, p. 647-663, 2006.

RAPP, A. O. Review on heat treatments of wood. In: SPECIAL SEMINAR HELD IN ANTIBES, FRANCE, 1., 2001, Brussels. Proceedings... Brussels: European Commission Research Directorate, 2001. p. 68.

SKAAR, C. Wood-water relations. New York: SpringerVerlag, 1988. 283 p.

TOMAZELLO FILHO, M.; SILVA, D. A. Formação e caracterização da madeira de compressão em Pinus caribaea var. hondurensis. IPEF, Piracicaba, n. 37, p. 51-59, 1987.

Cerne, Lavras, v. 19, n. 3, p. 391-398, jul./set. 2013 
UNSAL, O.; KORKUT, S.; ATIK, C. The effect of heat treatments on some properties and colour in Eucalyptus camaldulensis Dehn. wood. Maderas. Ciencia y Technologia, Concepcion, v. 5, p. 145-152, 2003.

WALKER, J. C. F. Primary wood processing: principles and practice. $2^{\text {nd }}$ ed. Christchurch: University of Canterbury, 2006. $596 \mathrm{p}$.
YEH, T. F. et al. Morphological and chemical variations between juvenile wood, mature wood, and compression wood of loblolly pine (Pinus taeda L.). Holzforschung, Berlin, v. 60, p. 1-8, 2006.

YILDIZ, S.; GEZER, E. D.; YILDIZ, U. C. Mechanical and chemical behavior of spruce wood modified by heat. Building and Environment, Oxford, v. 41, p. 1762-1766, 2006.

Recebido: 6 de abril de 2011; aceito: 20 de dezembro de 2012.

Cerne, Lavras, v. 19, n. 3, p. 391-398, jul./set. 2013 